Int. J. Morphol.,

30(3): 1158-1165, 2012

\title{
Peculiaridades Anatómicas del Canal Semicircular Superior y su Posible Implicancia Clínica
}

\author{
Anatomical Peculiarities of the Superior Semicircular Canals and its Possible Clinical Implication
}

"Jaime Whyte; ${ }^{* *}$ Claudio Martínez; *Ana Cisneros; ${ }^{* * *}$ Ana Whyte $\&{ }^{* * * *}$ Miguel Ángel Crovetto

\begin{abstract}
WHYTE, J.; MARTÍNEZ, C.; CISNEROS, A.; WHYTE, A. \& CROVETTO, M. A. Peculiaridades anatómicas del canal semicircular superior y su posible implicancia clínica. Int. J. Morphol., 30(3):1158-1165, 2012.

RESUMEN: El objetivo fue estudiar las peculiaridades en la morfología apical del canal semicircular superior, a nivel de la eminencia arcuata con la finalidad de obtener conclusiones aplicativas. Se estudiaron 48 cabezas de cadáveres humanos (96 huesos temporales), con la intención de identificar la presencia de adelgazamiento o dehiscencia del canal semicircular superior, así como la existencia de surcos vasculares de la eminencia acuata que, discurriendo sobre el techo del canal, pudieran afectar a su integridad anatómica. El estudio incluye un análisis histológico (Tricrómico de Martins) y radiológico (Tomografía Computarizada) de los hallazgos positivos (dehiscencias y adelgazamientos), así como estudio tomográfico de los canales con surcos vasculares visibles. En un caso $(1,04 \%)$ hemos observado cómo el canal presentaba un defecto óseo completo (dehiscencia), en tres $(3,12 \%)$ el canal presentaba una pared muy delgada cuyo grosor no excedía de $0,2 \mathrm{~mm}$. En 12 casos $(12,5 \%)$ la eminencia arcuata estaba recorrida por surcos por el que discurrían vasos colaterales meníngeos, que determinaron una concavidad entre 0,1 y $0,2 \mathrm{~mm}$, mientras que el grosor medio de dicha pared en estos casos fue de 1,2 mm. De las tres peculiaridades estudiadas, la falta de cobertura ósea es la que produce el síndrome de dehiscencia del canal semicircular superior. Los canales que presentaban una cubierta adelgazada pueden estar predispuestos a una rotura y por lo tanto causar patología en el canal. En cambio la presencia de surcos vasculares que recorren la superficie apical del canal, no parece tener consecuencias al disminuir minimamente su grosor.
\end{abstract}

PALABRAS CLAVE: Canal semicircular superior; Dehiscencia; Pared adelgazada; Surcos vasculares.

\section{INTRODUCCIÓN}

Los canales semicirculares superiores o anteriores se hallan situados en el espesor del peñasco del hueso temporal y forman parte del laberinto posterior del oído interno. Cada uno de ellos describe un arco que aproximadamente describe casi dos tercios de un círculo, y cuya longitud es de 15 a $20 \mathrm{~mm}$, su orientación puede ser oblícuo hacia arriba y afuera o generalmente vertical, existiendo grandes diferencias individuales.

Cada canal presenta dos extremos, uno dilatado (ampular), elíptico, con un diámetro de $3 \mathrm{~mm}$ y que desemboca en la porción superior y externa del vestíbulo; el otro extremo no ampular resultante de su unión con el del canal posterior, es circular con un diámetro de $1,5 \mathrm{~mm}$ y desemboca en la parte interna del vestíbulo.

El canal semicircular superior levanta por su bucle la cortical de la superficie antero-superior del peñasco formando un relieve transversal, la eminencia arcuata. Sin embargo esta eminencia no coincide exactamente con el canal semicircular óseo superior siempre. Para Faure et al. (2003) está eminencia sólo corresponde al relieve del canal semicircular superior en el $37 \%$.

Un aspecto importante del canal es el espesor de la lámina ósea que separa el canal de la fosa cerebral media, que para Tsunoda (2001) varía de 0,5 a 2,5 mm, con una media de $1,2 \mathrm{~mm}$ $( \pm 0,6)$ y para Crovetto et al. (2010) fue de 1,1 mm (DE 0,5).

Raramente esta cubierta está ausente, siendo entonces el canal dehiscente como describieron Minor et al. (1998) y Gianoli (2001), lo que determina en muchos de esos casos un síndrome clínico con sintomatología vestibular y auditiva.

Nuestro objetivo fue analizar las peculiaridades en la morfología apical del canal semicircular superior, a nivel de la eminencia arcuata.

\footnotetext{
* Departamento de Anatomía e Histología Humanas. Universidad de Zaragoza, Zaragoza, España.

** Hospital General de la Defensa. Servicio de Radiología, Zaragoza, España.

**** Departamento de Patología Animal. Universidad de Zaragoza, Zaragoza, España.

***** Hospital de Basurto. Servicio de Otorrinolaringología, Bilbao, España.
} 


\section{MATERIAL Y MÉTODO}

El estudio se realizó sobre cabezas de cadáveres formolados pertenecientes al osario del Departamento de Anatomía e Histología Humanas de la Facultad de Medicina de la Universidad de Zaragoza. Las muestras fueron recogidas entre los años 2005 y 2011.

En todos los cráneos se eliminó previamente la calota para acceder a la base del cráneo, se extrajo el cerebro y se disecaron bajo microscopio Zeiss OPMI las meninges, examinando a continuación la superficie de la eminencia arcuata, evaluando específicamente la existencia de adelgazamiento o dehiscencia del techo del canal semicircular superior.

En los casos en los que se observó la presencia de una dehiscencia, de una muy fina capa de hueso (pared translúcida),o de presencia de un surco que recorriera la eminencia arcuata, se procedió al estudio radiológico de los temporales mediante Tomografía Computarizada con un equipo Helicoidal Multicorte (Philips Brillance 6).

Los protocolos radiológicos para la adquisición y formateo de las imágenes han sido los siguientes: la colimación $2 \times 0,6 \mathrm{~mm}$, grosor de corte $0,65 \mathrm{~mm}$, incremento de corte $0,32 \mathrm{~mm}$, tiempo de rotación 0,75 segundos, pitch 0,38 , KV 120, mAs 300, matriz 1024 x 1024, campo de visión $180 \mathrm{~mm}$, grosor de reconstrucción $0,5 \mathrm{~mm}$ e incremento de reconstrucción $0,5 \mathrm{~mm}$. Se han efectuado reconstrucciones coronales y en el plano del canal semicircular superior en cada temporal. Los "datos crudos" se han reconstruido usando un algoritmo óseo.

Además en los casos que presentaron dehiscencia o pared translúcida se llevó a cabo una minuciosa y cuidada disección del canal en bloque, para a continuación descalcificarlos con acido nítrico al $3 \%$ a una temperatura de 25 grados centígrados. Después del proceso de descalcificación las muestras fueron procesadas para su inclusión en parafina, cortadas con un microtomo LEITZ a 9 micras y teñidas con la técnica del Tricrómico de Martins.

\section{RESULTADOS}

Hemos estudiado un total 48 cabezas de cadáveres (96 temporales), destacando los siguientes hallazgos:

Canal dehiscente. Uno de los casos (perteneciente a un sujeto varón de 72 años) presentó una dehiscencia del canal semicircular superior izquierdo. En la Figura 1a, la presencia de una solución de continuidad en el canal con una morfología elíptica alargada y márgenes lisos (flechas), su longitud máxima fue de 2,07 $\mathrm{mm}$ y la anchura $0,72 \mathrm{~mm}$. El resto del bucle del canal semicircular superior, aunque no mostraba dehiscencia, presentaba un adelgazamiento papiráceo de la pared hasta hacerlo translúcido.

En la Figura 1b se observa un corte histológico de una sección del mismo temporal izquierdo orientado en el plano coronal en el que destacamos el tejido óseo compacto que lo forma, la ausencia de hueso en la zona apical de éste (flecha), el adelgazamiento óseo progresivo en los límites de la dehiscencia con una distancia entre ambos extremos de $0,67 \mathrm{~mm}$.

La Tomografía Computarizada en el lado izquierdo mostró la ausencia de cobertura ósea en el techo del canal a nivel apical (flecha) tanto en el plano del canal (Fig. 1c) como en el coronal (Fig. 1d); su longitud fue de $2,07 \mathrm{~mm}$ y su anchura de $0,70 \mathrm{~mm}$, mientras que en el lado derecho el canal semicircular superior estaba íntegro.

Solamente hemos encontrado un caso de dehiscencia de canal semicircular superior en 96 temporales estudiados, lo que corresponde al 1,04\% de los oídos estudiados.

La correlación entre la morfología tanto macroscópica como microscópica y la tomografía computadorizada nos ha dado datos muy similares en cuanto a la longitud y la anchura de la dehiscencia.

Canal translucido. En tres casos ( 2 en adultos de 57 y 76 años respectivamente, y el tercero en un recién nacido) los canales semicirculares superiores presentaron una pared muy delgada translúcida que sigue el contorno del arco del canal (Figs. 2a, b y c), lo que corresponde al 3,12\% de los temporales estudiados.

En los cortes histológicos de los dos adultos (Fig. 2d) se aprecia una lámina ósea extremamente delgada y con un grosor para el primero de $0,17 \mathrm{~mm}$, de $0,20 \mathrm{~mm}$ para el segundo y de $0,18 \mathrm{~mm}$ para el tercero en la zona translúcida del canal, mientras que el resto de este presentaba grosores entre $0,6 \mathrm{~mm}$ y $1,2 \mathrm{~mm}$.

Siguiendo el protocolo realizamos Tomografía Computarizada y la posterior reconstrucción en el plano del canal donde se aprecia la integridad de la cubierta ósea constituida por una fina lámina ósea de una extrema delgadez cuyo grosor no excedía en ningún caso los $0,2 \mathrm{~mm}$ (Figs. 2e). 

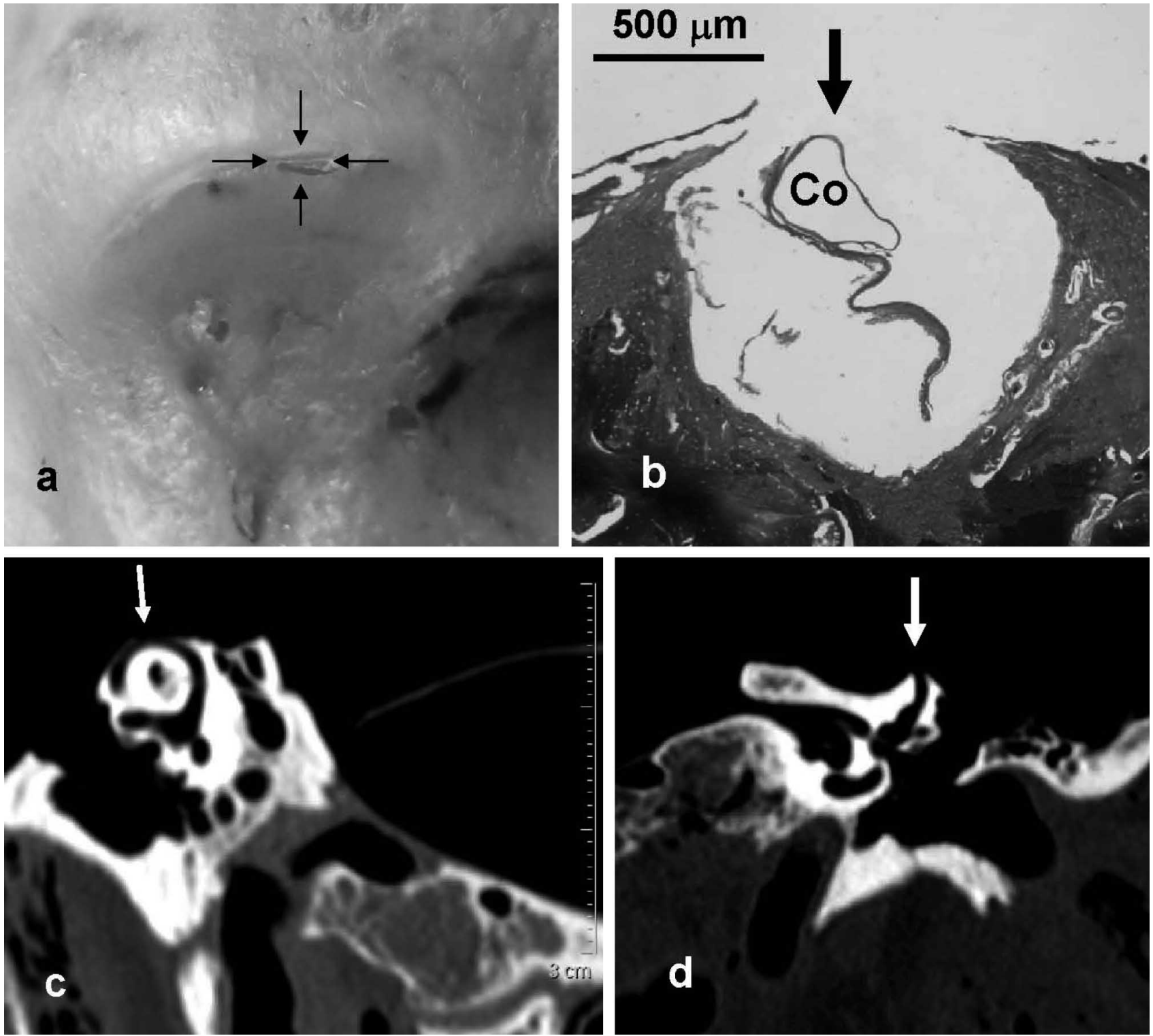

Fig. 1. Dehiscencia del canal semicircular superior (flechas). a) Visión macroscópica (flechas), b) corte histológico donde destaca el tejido óseo compacto del canal y la abertura a nivel de ésta (flecha), c y d) con tomografía computarizada en el plano del canal y coronal, donde se aprecia un defecto en la cobertura del canal. (b) Técnica del Tricrómico de Martins. Co: conducto semicircular).

Presencia de surcos que recorren el canal. En 12 temporales $(12,5 \%)$ hemos observamos al levantar la duramadre (D) como la eminencia arcuata estaba recorrida por un surco por el que discurrían vasos colaterales de las arterias meníngeas (A) (Fig. 3a).

La Figura 3 b muestra una pieza anatómica cuya eminencia está recorrida por un surco (flecha), y vemos como se diferencia claramente del canal del seno petroso superior $\left.{ }^{*}\right)$ que camina por el borde superior del peñasco.
Las reconstrucciones con Tomografías Computarizadas en el plano del canal mostraban un grosor medio de $1,17 \mathrm{~mm}$ a nivel de la lámina ósea, mientras que en el surco meníngeo la concavidad medía entre 0,1 y $0,2 \mathrm{~mm}$, con una media de $0,14 \mathrm{~mm}$ (Tabla I).

Nótese en la Figura 3c una panorámica y un detalle del canal semicircular donde destaca la presencia de dos celdillas aéreas y entre ellas el pequeño canal (flecha) así como la muesca que en el canal deja el seno petroso cuyo grosor en este caso fue de $1,18 \mathrm{~mm}(*)$. 

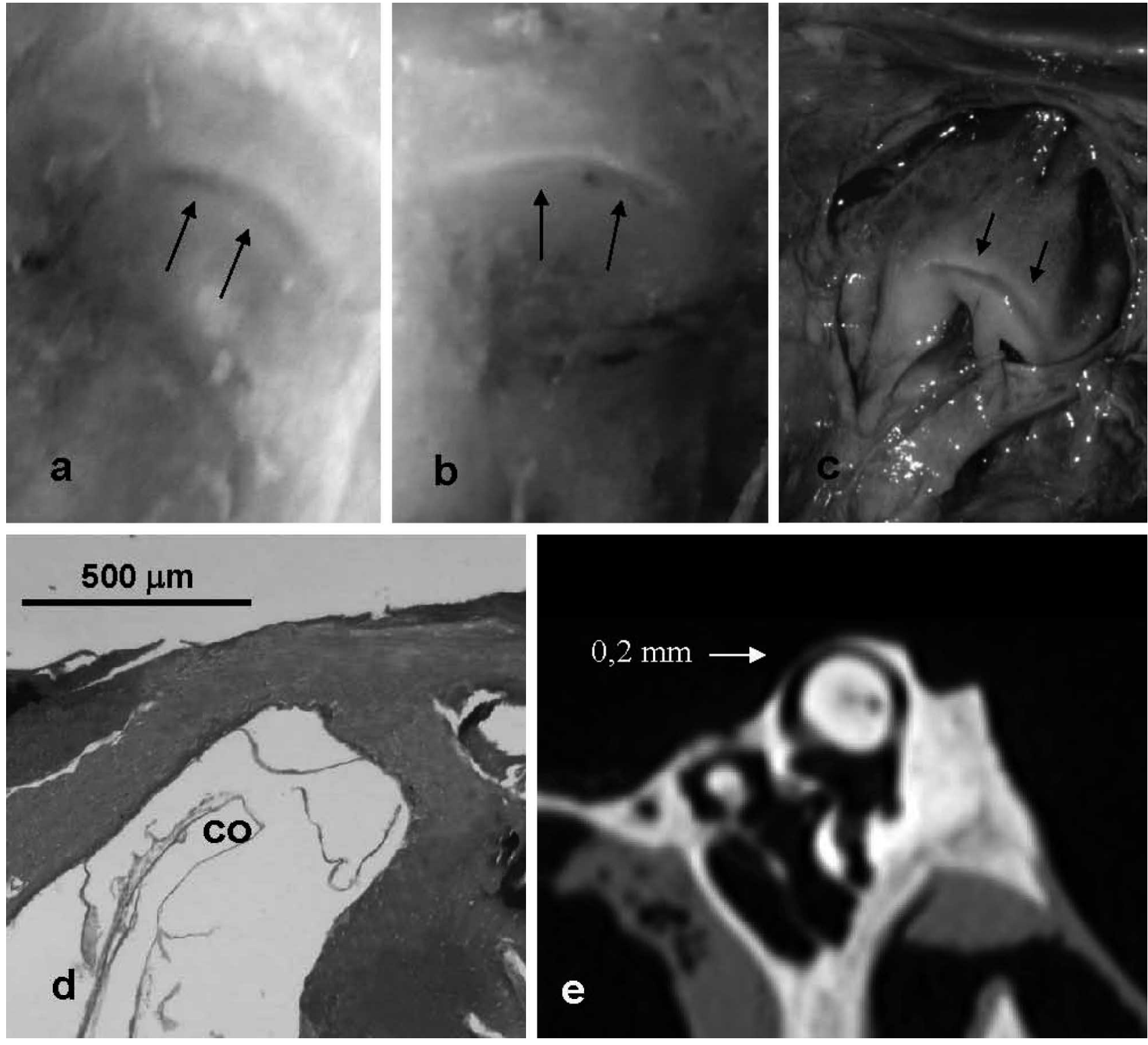

Fig. 2. Canal semicircular superior translúcido. Nótese la integridad de la cubierta ósea y su extrema delgadez. a, b y c) Visión macroscópica (flechas), en tres casos de 57, 76 años y recién nacido respectivamente, d) microscópica donde se observa a nivel apical el adelgazamiento de la cubierta ósea del canal y e) con tomografía computarizada en el plano de canal dicho adelgazamiento (flecha). (b) Técnica del Tricrómico de Martins. Co: conducto semicircular)

Tabla I. Relación entre el gro-

\begin{tabular}{ccc}
\hline CASOS & Medida PARED de CSS & Medida de SURCO \\
\hline 1 & 0,9 & 0,1 \\
2 & 1 & 0,1 \\
3 & 1,2 & 0,2 \\
4 & 1,3 & 0,2 \\
5 & 1,6 & 0,1 \\
6 & 0,8 & 0,1 \\
7 & 1 & 0,2 \\
8 & 1,5 & 0,1 \\
9 & 1,4 & 0,1 \\
10 & 1,2 & 0,1 \\
11 & 1,8 & 0,2 \\
12 & 1,4 & 0,2 \\
\hline
\end{tabular}



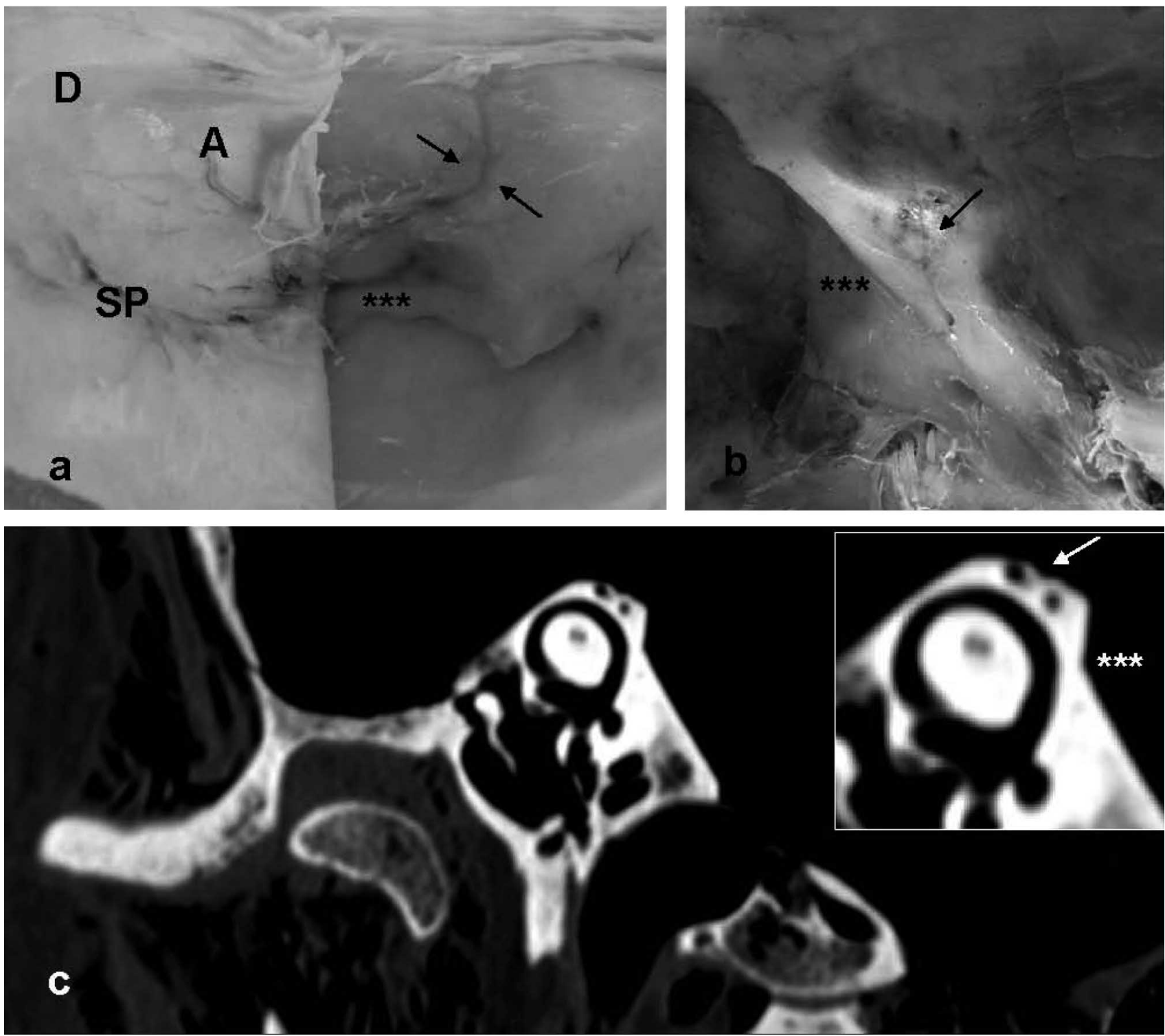

Fig. 3. Nótese la presencia de surcos en relación con el canal semicircular superior. a y b) imágenes macroscópicas y c) con tomografía en el plano de canal en donde se señala con una flecha el surco vascular. (Flechas) surco meníngeo, (***) surco seno petroso superior, (A) vaso meníngeo, (SP) seno petroso superior, (D) duramadre.

\section{DISCUSIÓN}

En el estudio del canal semicircular superior en cadáveres humanos hemos observado tres peculiaridades en su morfología. La primera encontrada es la presencia de un defecto óseo completo (dehiscencia) localizado en el arco del canal semicircular superior, que hemos encontrado en un único caso. La dehiscencia del canal semicircular superior es un síndrome hoy día bien establecido como una forma de vestibulopatía periférica que puede cursar con alteraciones vestibulares y/o auditivas en unos casos, y en otros ser completamente asintomático (Hillman et al., 2006), habiendo indicios clínicos que relacionan la presencia de sintomatología vestibular y auditiva con el tamaño de la dehiscencia (Pfammatter et al., 2010).

Gianoli la define como la ausencia de cobertura ósea sobre este canal. Este hecho conlleva que su luz interior quede anormalmente abierta al espacio intracraneal, en contacto directo con la meninge que tapiza el suelo de la fosa media. 
En la actualidad, a pesar de la resolución de los métodos de diagnóstico por imagen, existen discrepancias sobre la prevalencia real de dicho síndrome ya que los estudios radiológicos nos indican porcentajes marcadamente superiores que los anatómicos. Así mediante medidas realizadas sobre CT de temporales, Crovetto et al. encuentran una incidencia del 3,6\% en su serie de 604 temporales, cifra que llega al $4 \%$ en la serie de Cloutier et al. (2008) y al $9 \%$ en el estudio de Williamson et al. (2003) encontrando otros autores incidencias incluso superiores (Ceylan et al., 2010; Piton et al., 2008). En cambio en estudios realizados post-mortem $\mathrm{y}$ en piezas de osario se calcula que el porcentaje de sujetos afectos de este tipo de dehiscencia en la zona superior del canal es aproximadamente de 0,5\% para Carey et al. (2000), y para Tsunoda \& Terasaki (2002) del $0,4 \%$ en huesos aislados y del 1,4 \% (1 caso de 69) en cadáveres. Nosotros hemos observado en nuestro estudio anatómico realizado con temporales de cadáveres una incidencia del 1,04 \% (1 caso de 48).

Estos datos nos indican cómo la prevalencia de los estudios radiológicos es marcadamente superior que la de los estudios anatómicos. Esta discrepancia pensamos que es debida a que la resolución de las Tomografías Computarizadas no es suficiente para delinear hueso muy fino, o por la orientación de los cortes del canal en el plano coronal (Crovetto et al.).

Masaki (2011) afirma que cuando el diagnóstico de síndrome de dehiscencia se hizo exclusivamente a base de tomografía, el $80 \%$ de los casos evaluados fueron falsos positivos. Este autor propone que se debe llegar siempre a la clasificación del Síndrome a partir de la existencia de sintomatología clínica y diagnóstico tomográfico, en cuyo caso la incidencia en su estudio de 164 temporales desciende del $3 \%$ (5 casos) al 0,6\% ( 1 caso).

El estudio histológico del único caso de dehiscencia del canal semicircular superior que hemos encontrado en nuestra serie nos permite observar la falta de una parte de la cubierta ósea y cómo el canal queda abierto hacia la fosa cerebral media, pero hay que tener en cuenta que para su observación macroscópica hemos tenido que disecar la duramadre que en condiciones normales está ocluyendo a dicha abertura.

Nosotros pensamos que la duramadre tiene que jugar un papel importante ya que se comporta como un verdadero periostio y ocluiría por tanto la dehiscencia, y así se explicaría porqué algunos pacientes son asintomáticos y porqué los síntomas no están presentes hasta edades avanzadas que es cuando la duramadre aumenta de tamaño y se hace fibrosa. De otro lado, como estudió Greenwald et al.
(2000), la duramadre en animales jovenes elabora factores de crecimiento osteogénico (TGF.beta33) y matriz extracelular (colágena tipo III), lo que promueve la osificación del cráneo. Esto podría explicar porque en la infancia y en la edad juvenil no se manifiesta clínicamente la dehiscencia del canal semicircular superior, ya que la vitalidad osteogénica de la duramadre mantiene cerrada la dehiscencia. Posteriormente la pérdida de vitalidad de la duramadre hace patente fisiopatológicamente la dehiscencia.

La segunda característica del canal que queremos reseñar es la de aquellos canales que macroscópicamente presentaban una cubierta papiracea del canal a nivel de la fosa media, mostrándose en la visión directa como una línea translucida en la eminencia arcuata.

Hemos observado en tres cadáveres la presencia de una fina capa de hueso cubriendo el canal semicircular superior cuyas medidas fueron en los tres casos igual o inferiores a $0,2 \mathrm{~mm}$. Considerando que el grosor medio del hueso que cubre el canal semicircular superior fue de 1,1 (DE $0,5)$, y para Crovetto et al. a 1,2 (DE 0,6) para Tsunoda \& Terasaki, resulta cierta la afirmación de que los tres casos presentados tienen coberturas óseas del canal semicircular superior muy adelgazadas. El porcentaje del hallazgo afecta al 3,12 \% de los casos estudiados por nosotros, cifra superior a la aportada por Carey et al. que describe dicho adelgazamiento en el 1,3\% de su serie de 1000 temporales, aunque para este autor el límite fijado para determinar un adelgazamiento de la cobertura osea era de $0,1 \mathrm{~mm}$. La diferencia de casuística entre el estudio de Carey et al. y el nuestro también podría explicar las diferencias porcentuales encontradas entre series.

Pensamos que esta disminución de grosor podría hacer que con un suceso como un traumatismo craneal, un brusco aumento de la presión intracraneal o la erosión causada por el peso y presión del lóbulo temporal, se produjera su ruptura y la posterior dehiscencia (Watters et al., 2006; Ogutha et al., 2009; López-Vázquez et al., 2011).

La presencia en el canal de una zona que presenta menor grosor la interpretamos como la banda ósea resultante de la fusión con los centros de osificación canaliculares superior e inferior del conducto semicircular superior en su desarrollo, como sugiere el caso del recién nacido donde se aprecia la fusión de los centros preexistentes.

El no haber encontrado en todos los peñascos una zona translucida tendría su explicación en que después del nacimiento el canal se robustece paulatinamente por adición de hueso perióstico en sus superficies exteriores (SmithAgreda et al., 1985). 
El tercer punto era ver si la presencia de surcos vasculares podría influir en el adelgazamiento de la pared ósea del canal y causar patología. Los dos lugares donde con mayor frecuencia aparecen dehiscencias son donde el seno petroso superior está en contacto con el canal (Carey et al.), y en la parte superior o apical del canal (Crovetto et al.) donde ambas zonas tienen relación con los surcos vasculares.

La disección de la eminencia arcuata nos ha dejado ver la presencia en 12 temporales de surcos que recorren la superficie apical del canal, por donde caminaban los vasos meníngeos con un porcentaje del 12,5\%.

Mediante tomografía hemos medido estos surcos meníngeos con un valor máximo de $0,2 \mathrm{~mm}$, y como de- muestra la iconografía modifican muy poco el grosor de la cubierta ósea, y por lo tanto no producen grandes erosiones en su pared y creemos que no influyen en las posibles causas de producción de una dehiscencia.

En cuanto al canal del seno petroso no hemos observado en ninguno de los casos estudiados disminución de la pared del canal, hecho similar al descrito por Minor (2000) que no encuentra en Tomografía Computarizada de alta resolución dehiscencia o adelgazamiento confinado al seno petroso superior. Pensamos por lo tanto que dicho canal petroso no influye en las causas etiológicas de dehiscencia aunque Puwanarajah et al. (2008) comunicara un caso en el que el seno petroso superior estaba agrandado y recibía el drenaje de una gran vena cerebelosa anómala, presentando dehiscencia del canal semicircular superior a este nivel.

WhYTE, J.; MARTínez, C.; CISNEROS, A.; WHYTE, A. \& CROVETTO, M. A. Anatomical peculiarities of the superior semicircular canals and its possible clinical implication.Int. J. Morphol., 30(3):1158-1165, 2012.

SUMMARY: The aim was to study the peculiarities in apical morphology of the superior semicircular canal are studied at level of the arcuate eminence to obtain application conclusions. We have studied 48 heads from human cadavers ( 96 temporal bones), with the intention of identifying the presence of thinning or dehiscence in the superior semicircular canal, as well as the existence of vascular sulci of the arcuate eminence, which, traveling along the roof of the canal, may affect its anatomical integrity. The study includes a histological (Martin's Trichome method) and a radiological analysis (Computerized Tomography) of the findings (dehiscences and thinnings), and as well as tomographic study of the canals with visible vascular sulci. In one case $(1.04 \%)$ we have observed how the canal presented a complete bone defect (dehiscence), in three (3.12\%), presented a very thin wall, which did not exceed $0.2 \mathrm{~mm}$ thick. In $12 \mathrm{cases}(12.5 \%)$ sulci covered the arcuate eminence through which meningeal collateral vessels ran, which determined a concavity of between 0.1 and 0.2 $\mathrm{mm}$, whilst the average thickness of this wall in these cases was $1.2 \mathrm{~mm}$. Of the peculiarities studied, the lack of bone coverage is what produces the dehiscence syndrome of the superior semicircular canal. The canals that had a reduced coverage may be predisposed to breakage and therefore cause pathology in them. On the other hand, the presence of vascular sulci that ran along the apical surface of the canal seems to have no consequences to decrease their thickness.

KEY WORDS: Superior semicircular canal; Dehiscence; Thinned wall; Vascular furrows.

\section{REFERENCIAS BIBLIOGRÁFICAS}

Carey, J. P.; Minor, L. B. \& Nager, G. T. Dehiscence or thinning of bone overlying the superior semicircular canal in a temporal bone survey. Arch. Otolaryngol. Head Neck Surg., 126(2):13747, 2000.

Ceylan, N.; Bayraktaroglu, S.; Alper, H.; Savas, R.; Bilgen, C.; Kirazli, T.; Güzelmansur, I. \& Ertürk, S. M. CT imaging of superior semicircular canal dehiscence: added value of reformatted images. Acta Otolaryngol., 130(9):996-1001, 2010.

Cloutier, J. F.; Bélair, M. \& Saliba, I. Superior semicircular canal dehiscence: positive predictive value of high-resolution CT scanning. Eur. Arch. Otorhinolaryngol., 265(12):1455-60, 2008.

Crovetto, M.; Whyte, J.; Rodriguez, O. M.; Lecumberri, I.; Martinez, C. \& Eléxpuru, J. Radiological study of the superior semicircular canal dehiscence. Radiological considerations of superior and posterior semicircular canals. Eur. J. Radiol., 76(2):167-72, 2010.

Faure, A.; Masse, H.; Gayet-Delacroix, M.; Khalfallah, M.; Bordure, P.; Hamel, O.; Raoul, S.; Leborgne, J. \& Robert, R. What is the arcuate eminence? Surg. Radiol. Anat., 25(2):99104, 2003.

Gianoli, G. J. Deficiency of the superior semicircular canal. Curr. Opin. Otolaryngol. Head Neck Surg., 9:336-41, 2001.

Greenwald, J. A.; Mehrara, B. J.; Spector, J. A.; Fagenholz, P. J.; Saadeh, P. B.; Steinbrech, D. S.; Gittes, G. K. \& Longaker, M. T. Immature versus mature dura mater: II. Differential expression of genes important to calvarial reossification. Plast. Reconstr. Surg., 106(3):630-8, 2000.

Hillman, T. A.; Kertesz, T. R.; Hadley, K. \& Shelton, C. Reversi- 
ble peripheral vestibulopathy: the treatment of superior canal dehiscence. Otolaryngol. Head Neck Surg., 134(3):431-6, 2006.

López-Vázquez, A.; Carmen Sampériz, L.; Matallana Barahona, S.; Serrano Añon, E.; Artal Sánchez, R.; Agreda Moreno, B. \& Valles Varela, H. Dehiscencia del canal semicircular superior. A propósito de un caso. ORL Aragón, 4(1):18-9, 2011.

Masaki, Y. The prevalence of superior canal dehiscence syndrome as assessed by temporal bone computed tomography imaging. Acta Otolaryngol., 131(3):258-62, 2011.

Minor, L. B. Superior canal dehiscence syndrome. Am. J. Otol., 21(1):9-19, 2000.

Minor, L. B.; Solomon, D.; Zinreich, J. S. \& Zee, D. S. Soundand/or pressure-induced vertigo due to bone dehiscence of the superior semicircular canal. Arch. Otolaryngol. Head Neck Surg., 124(3):249-58, 1998.

Ogutha, J.; Page, N. C. \& Hullar, T. E. Postpartum vertigo and superior semicircular canal dehiscence syndrome. Obstet. Gynecol., 114(2 Pt 2):434-6, 2009.

Pfammatter, A.; Darrouzet, V.; Gärtner, M.; Somers, T.; Van Dinther, J.; Trabalzini, F.; Ayache, D. \& Linder, T. A superior semicircular canal dehiscence syndrome multicenter study: is there an association between size and symptoms. Otol. Neurotol., 31(3):447-54, 2010.

Piton, J.; Negrevergne, M. \& Portmann, D. Dehiscence of the superior semicircular canal: approach and CT scan classifications. Rev. Laryngol. Otol. Rhinol. (Bord), 129(1):17-26, 2008.

Puwanarajah, P.; Pretorius, P. \& Bottrill, I. Superior semicircular canal dehiscence syndrome: a new aetiology. J. Laryngol. Otol., 122(7):741-4, 2008

Smith-Agreda, V.; Ferres Torres, E. \& Montesinos Castro-Girona, M. Atlas-Tratado de Anatomía del Sistema Nervioso con introducción a la clínica y EEG. Valencia, Ed. Gregori, 1985.

Tsunoda, A. Arcuate eminence in Caucasian populations. $J$. Laryngol. Otol., 115(1):9-13, 2001.

Tsunoda, A. \& Terasaki, O. Dehiscence of the bony roof of the superior semicircular canal in the middle cranial fossa. $J$. Laryngol. Otol., 116(7):514-8, 2002.

Watters, K. F.; Rosowski, J. J.; Sauter, T. \& Lee, D. J. Superior semicircular canal dehiscence presenting as postpartum vertigo. Otol. Neurotol., 27(6):756-68, 2006.

Williamson, R. A.; Vrabec, J. T.; Coker, N. J. \& Sandlin, M. Coronal computed tomography prevalence of superior semicircular canal dehiscence. Otolaryngol. Head Neck Surg., 129(5):4819, 2003.

\author{
Dirección de corespondencia: \\ J. R. Whyte Orozco \\ Departamento de Anatomía e Histología Humanas. \\ Facultad de Medicina \\ Universidad de Zaragoza \\ $\mathrm{C} /$ Domingo Miral, s/n, \\ 50009 Zaragoza \\ ESPAÑA
}

Email: jwhyte@unizar.es

Recibido : 02-03-2012

Aceptado: 15-05-2012 